\title{
电力系统设备状态监测与故障诊断技术分析
}

\section{Technical Analysis of Power System Equipment Condition Monitoring and Fault Diagnosis}

曾令文

\author{
Lingwen Zeng
}

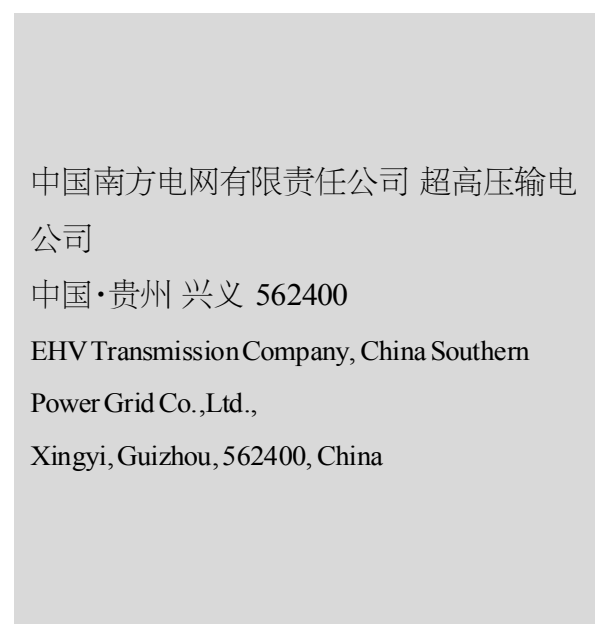

\section{1 引言}

对于电力系统设备来说, 无论是状态监测还是故障诊断, 基本上都是一类全新的技术, 以此确保电力系统能够更好地 完成运行工作。因此,相关人员便需要提高重视程度, 针对这 一方面进行深人研究。

\section{2 状态监测和故障诊断技术的基本概念}

基于系统设备本身,在应用监测技术的时候,主要应用的 便是传感器, 并应用一些最新的测量方式, 从而能够得到相关 数据资料。对于这些数据来说, 可以有效反映出设备现阶段的 工作情况, 以此能够有效把握设备的运行质量, 是否处于正常 的状态, 是否有任何故障问题存在。

对于设备故障诊断工作来说, 主要是基于其当前的影响 状态, 以此获得相关数据内容, 通过和正常运行时的数据展开 对比, 能够推断出故障的种类以及出现的具体原因。一般来 说, 主要是基于特征量本身展开深人分析, 进而明确状态监测 的内容。

\section{3 状态监测的相关技术}

\section{1 信号采集}

设备在进行监测的时候, 首要工作任务便是有效把握当 前诊断对象的基础信息, 以此得到设备的相关电信号。基于其 状态量的差异化特色,选择不同的诊断方式。一般来说, 具体 采样的方法主要为四类: 其一是一次性处理, 每一次采集时,
仅仅采集一个实际长度满足处理需求的信号即可; 其二是基 于规定的时间要求进行处理; 其三是结合当前出现的故障情 况,在第一时间发出信号, 以此展开采样; 其四是基于故障的 基本要求,采取一些针对性方式进行采样。

对于不同的设备来说, 相关任务要求完全不一样,因此, 应用的监测方式也有着诸多差异。但凡变压器出现了任何问 题, 基本上都是因为内部材料出现了老化的情况, 因此, 便需 要结合变压器本身的特点,采用局部放电的方式展开分析。而 对于电机来说, 每次出现的故障类别都有着诸多区别, 而且原 因也有很多种。因此,便可以尝试采用神经网络的方式展开预 测。在针对断路器的状况进行诊断时, 最为常用的方式便是跳 闸轮廓法,以此能得到设备现阶段的信息资料。

\section{2 数据传送}

通常来说, 主要负责信号处理的系统往往会和监测设备 之间有着较大的距离。因此, 在进行数据传输的时候, 很容易 受到外部因素的破坏。所以,工作人员便需要针对数据资料进 行处理。首先是展开 $\mathrm{A} / \mathrm{D}$ 转化, 在完成数据压缩之后, 对其进 行打包, 最后再全部都传到控制中心内部。目前来说, 在中国 当前电力领域之中, 通讯设备的应用率越来越高, 通过采用光 纤传输数字信号, 可以有效处理外部干扰造成的影响[1]。

\section{3 数据处理}

内部中心在得到数据之后, 通常会通过多种数学方案展 开处理。如在频谱分析的过程中,可以尝试将时域连续时间信 号转化为频域不同的信号。不仅如此, 最为常用的分析方式还 
故障分析 Fault Analysis

包括神经网络以及小波分析等。在实际处理的过程之中,可以 通过数字技术方式, 并将其和智能技术结合在一起, 以此完成 数据资料的处理工作,进而提升精确性。

\section{4 故障诊断的相关技术}

\section{1 合理选择信号的特征量}

在现有的所有能够进行正常使用功能的信息资料中, 将 最为重要的内容提取出来,这便是信号处理的主要工作。当设 备在实际运行时, 如果提供的数据资料更具精确性特点, 那么 在进行故障沴断时,整体灵敏度便会得到提高。对于任何故障 特征量来说, 绝对不是由单一问题造成的。然而通常来说, 通 过对于这些特征量展开深入分析，可以发现其基本上直接代 表着某些特定的故障问题。如果工作人员未能有效明确特征 量, 自然无法对设备的运行情况予以把握, 了解其当前究竟是 出现了故障还是正常运行。毕竟即便当设备在正常运行时,整 体表现同样和故障情况存在相同的部分, 因此,便会让工作人 员做出错误的判断。正是这一情况, 理应在方法层面做出最为 正确的决定 ${ }^{[2]}$ 。

\section{2 故障诊断工作}

对于电力设备来说,自身出现故障的种类有很多种。即便 出现了同一类事故情况,往往也是由多种不同的状态共同展现 出来的。因此, 在进行诊断时, 主要可以从三个方面进行选择。

其一是部分故障会由多种情况表现出来, 因此,便需要通 过传感技术的方式展开判断。面对同一问题，从多个方面人 手,并使用各类不同的传感器展开处理,从而能够基于不同的 特征表现, 从中选择一类整体灵敏度更高的信息量,进而确保 决策判断的合理性。

其二是特征空间矢量法, 首先针对故障问题的相关数据 进行分析, 并展开深入学习。之后再通过不同的方法, 对其展 开修正。一般来说,所有故障对象往往都存在着慢时变性以及 不确定性的基本特点。

其三是通过人工智能技术的应用，以此保证技术分析的 方式更具科学性。相比于工作人员自主判断, 人工智能分析问 题自然更为全面, 往往能够看到工作人员无法看到的内容。

\section{3 信息技术以及故障分析技术}

对于故障分析技术来说, 主要是针对设备展开深人了解, 基于故障产生的实际情况,分析故障出现的具体原因。其一是 针对当前设备的特征量以及降维进行总结, 以此采用一些识 别类技术, 从而能够有效计算出具体参数内容, 进而可以得出 问题产生的主要因素。对于现有的设备来说,基本上都有了网 络传输的基本能力,因此,工作人员也可以通过信息传输的方
式进行判断。如果问题较为严重, 还可以通过远程协助, 向其 他人员求助, 以此共同展开问题诊断。如此一来, 设备状态的 判断将会更为精确。除此之外, 工作人员还可以尝试应用虚拟 诊断的设备, 将当前得到的实时数据上传到网络平台之中。

\section{5 当前现状以及未来发展}

目前,对于系统状态的监测工作来说,基本上已经在欧美 国家得到了全面普及。特别是在美国, 技术发展特别快, 几乎 所有电力企业内部都无需人工展开检修, 设备能够自主完成 处理工作。

在电力行业之中, 无论是状态检测还是故障沴断, 均取得 了非常好的效果。结合国外某些工厂提供的数据资料可以得 知, 在应用了人工智能技术后, 事故率大幅度下降, 维修率同 样有所减少。如此一来,企业便无需投入大量的资金成本进行 维修,进而提升了企业自身的经济效益。

自从 1980 年开始, 中国便已经有了这方面的相关研究。 因此,比起国外企业, 整体发展并不算晚。在经过了 30 多年 后, 中国已经有了独立自主研发的传感器。当前诸多工厂内部 都应用了这一设备,在线监测技术也得到了全面普及,从而使 得监测质量以及管理效果得到了提高。一些经济条件较好的 企业,开始从国外进口, 并将其和计算机技术结合在一起, 以 此创造了全新的监测系统, 能够有效把握设备的运行质量。

然而, 在与国外进行对比之后能够发现, 中国传感器的技 术水平仍然较为落后。不仅许多企业并未意识到技术监测的 意义所在, 而且软环境和管理体制均有着诸多缺陷。造成这一 问题出现的主要原因便是许多企业并未觉得监测技术有着很 高的应用价值, 因此, 在检修时, 仍然在应用传统的技术。另 外, 有些企业内部虽然已经有了相关设备, 但是工作人员并未 掌握这一方面的技术, 同时个人经验也不足, 从而造成技术资 源被完全浪费。

\section{6 结语}

综上所述, 对于电力企业来说, 系统监测工作越来越重 要。因此, 相关人员便需要提高重视程度, 结合设备正常运行 的状况, 对其展开把握, 诊断其中可能出现的问题, 并提前进 行预案。如此一来, 设备的整体水平必然会得到提高, 进而能 够有效推动中国电力行业得到全面发展。

\section{参考文献}

[1]苏鹏声,王欢.电力系统设备状态监测与故障沴断技术分析 [J]. 电力系统自动化,2018,27(1):61-65.

[2]李强,牛帅奇. 电力系统设备状态监测与故障诊断技术分析 [J]. 科技传播,2019,4(20):61-65. 